\title{
Childhood tuberculosis: a concern of the modern world
}

\author{
Agnes Hamzaoui ${ }^{1}$ Sadok Yaalaoui ${ }^{2}$, Fatma Tritar Cherif $^{3}$, Leila Slim Saidi ${ }^{4}$ \\ and Anissa Berraies ${ }^{1}$
}

Affiliations: 'Dept of Paediatric Respiratory Diseases, Abderrahmen Mami Hospital, Ariana, Tunisia.
${ }^{2}$ Immunology Laboratory, Abderrahmen Mami Hospital, Ariana, Tunisia. ${ }^{3}$ Dept of Respiratory Diseases C,
Tuberculosis Multidrug Resistance Unit, Abderrahmen Mami Hospital, Ariana, Tunisia. ${ }^{4}$ Microbiology
Laboratory, Mycobacterial Unit, Abderrahmen Mami Hospital, Ariana, Tunisia.

Correspondence: Agnes Hamzaoui, Dept of Paediatric Respiratory Diseases, Unit Research $15 S P 12$, Abderrahman Mami Hospital, El Manar Tunis University, 2080 Ariana, Tunisia.

E-mail: agnes.hamzaouidgmail.com

0

@ERSpublications

Childhood tuberculosis is a potentially lethal disease, which is underdiagnosed and undertreated http://ow.ly/yTJyt

As childhood tuberculosis (TB) reflects recent transmission, its burden provides an accurate measure of the level of TB control achieved in a particular community. Moreover, infected children represent the main reservoir of Mycobacterium tuberculosis (MTB) as potential future cases. However, childhood TB is neglected by scientists, policy makers, healthcare professionals and product developers [1]. Moreover, the interests of individual patients and public health may be conflicting. As children are considered in the majority of cases as noncontagious, asymptomatic disease is frequently ignored [2]. This is why, in 2013, the World Health Organization (WHO) developed a roadmap aiming to achieve zero deaths due to childhood TB by 2025 [3]. This article aims to highlight the underestimated reality of childhood TB, emphasising improved diagnosis possibilities and new treatment modalities, and advocating for dedicated paediatric operational research and clinical trials.

\section{Methods}

Active scanning of the recent literature using the keywords: "children tuberculosis", "latent tuberculosis infection", "new diagnosis tools" and "treatment modalities" was performed using PubMed and EMBASE. In addition, International Union Against Tuberculosis and Lung Disease publications were screened and WHO policy and guidance documents on TB were obtained from the WHO website (http://www.who.int).

\section{Epidemiology}

Each year, more than 74000 children die from TB [3]. Exposure to an adult with pulmonary TB was reported to increase mortality by $70 \%$ in children under 5 years of age in high-burden settings and by eightfold when the mother had TB [4].

The global burden of childhood TB is under-reported due to paucibacillary disease and the difficulty of confirming the diagnosis. In China, the prevalence rates for bacteriologically positive pulmonary $\mathrm{TB}$ and smear-positive cases were eight and 13 times less than the clinically diagnosed pulmonary $\mathrm{TB}$ rate, respectively [5]. TB disease and latent TB infection (LTBI) frequencies in children are dramatically underestimated. According to national reports, childhood TB accounted for $3.5 \%$ of the global TB caseload

Received: June 132014 | Accepted after revision: July 012014

Conflict of interest: None declared.

Provenance: Submitted manuscript, peer reviewed.

Copyright OERS 2014. ERR articles are open access and distributed under the terms of the Creative Commons Attribution Non-Commercial Licence 4.0. 
in high-burden countries, while best estimates suggest that $11 \%$ of TB cases occur in children, with 332000 paediatric patients being undiagnosed or unreported $[2,6]$. This data discrepancy also concerns Western Europe. An active surveillance system demonstrated an underestimation of childhood TB in the UK by about one-fifth [7]. The cumulative risk of infection in Greenland at 18 years of age was $13.4 \%$ [8].

Children's risk factors for infection are younger age, birth in a high prevalence country or foreign-born parents, HIV infection and prolonged close contact with the index case. Related index case risk factors are smoking, being a young adult, having cavitary lesions and a positive sputum smear test [9, 10]. Smearnegative and culture-positive patients, extrapulmonary TB cases and TB among schoolmates, while less contagious, also constitute a source of contamination $[4,6,11-13]$. The period of time in close contact with the TB patient is essential. When the index case was a teacher, the relative risk of exposed children, at school for $>6$ h per day, was approximately $30[9,14]$. By contrast, a brief exposure to a highly infectious individual may not result in a high risk for healthy children [15]. A contact-scoring tool estimated exposure quantification by considering maternal TB and sleep proximity, index case infectivity, duration of exposure and exposure to multiple index cases. It allowed detection of $80 \%$ of a child's risk of infection in a highburden setting [16]. In South African townships the mean number of smear-positive adults per exposed child for uninfected children, latently infected children and TB cases was 1, 1.6 and 1.9, respectively [17]. The majority of childhood TB cases were diagnosed in the same year as an adult index case. However, more than $30 \%$ of the time the adult case was diagnosed later, demonstrating the ongoing contamination in the community [17]. In a high endemicity setting, exposure to a known TB case in the past 2 years did not increase TB risk, while biomass exposure was a significant risk factor [18]. In high-burden TB communities, the first infection occurs in childhood, but is not a single lifetime event [19].

Family clusters of TB cases are ascribed to genetic susceptibility. Within the same house, TB mortality risk was higher for children belonging to the index family than for children living there who were not related to the index [4]. In Greenland, annual risk of TB infection was independent of age and bacilli Calmette-Guérin (BCG) vaccination programme, but varied substantially by ethnicity [8].

Disease risk after primary infection with MTB is greatest in infants and declines to a nadir at age 5-10 years. In Western Europe more than half of children with TB are $<5$ years old [20]. Endogenous reactivation might account for the second peak of TB in adolescence $[21,22]$. A temporal association has been reported between children's hospitalisations for TB, invasive pneumococcal diseases and influenza [23].

HIV infection has had a large impact on the epidemiology and severity of childhood TB [6]. In a prospective study conducted in the Western Cape, 23.4 cases of active TB were recorded per $100 \mathrm{HIV}$-infected children per year [24]. The high TB incidence among HIV-infected children is, in part, explained by increased risk of TB exposure [25]. The available epidemiological data for TB in childhood are summarised in table 1.

\section{Natural history: LTBI and immunological data}

Until recently, LTBI was supposed to precede TB disease, implying persistence of a low number of dormant mycobacteria in the organism. However, in numerous cases infection is a self-limited disease with no risk of progression [35]. In addition, in recently exposed children, the boundary line between primary TB and LTBI may be difficult to define [36]. Smear positivity in some asymptomatic and chest radiographynegative children further blurs the boundaries between infection and disease. The risk of progression to TB is highest after a median incubation period of 6 weeks [37]. The physical location of bacilli during latent infection remains poorly understood [35]. The persistent bacilli are thought to encounter constraining conditions that induce a distinct set of gene expression profiles and chromosomal mutations [38].

Innate immunity constitutes a strong line of defence against MTB. These innate response mechanisms may be powerful enough to prevent an adaptive specific response [39, 40]. The T-cell population, which is essential to overcome acute infection, is mainly made up of T-helper (Th)1, Th17, cytotoxic T-cells and regulatory T-cells [35]. Th1 differentiation is induced by interleukin (IL)-12 and IL-18. Th1 cells produce interferon (IFN)- $\gamma$, which in conjunction with tumour necrosis factor (TNF)- $\alpha$ activates macrophages and modulates granuloma constitution [39]. The balance of T-cell subpopulations at the level of the granuloma regulates protective immunity, inflammatory pathways and anti-inflammatory responses [35, 41]. B-cells are observed in significant numbers in granulomas, but their role remains undefined [40].

Secretion of IFN- $\gamma$ by lymphocytes is a poor correlate of protection as it depends on the antigenic load [35]. In vitro, T-cells from infected children stimulated by mycobacterial antigens produce Th1 and Th2 cytokines with no differences between LTBI and TB disease cases [42]. The frequencies of MTB specific CD4, CD8 and $\gamma \delta$ T-cells in blood do not correlate with vaccination protection in 10-week-old children who have received routine infant vaccination [43]. 


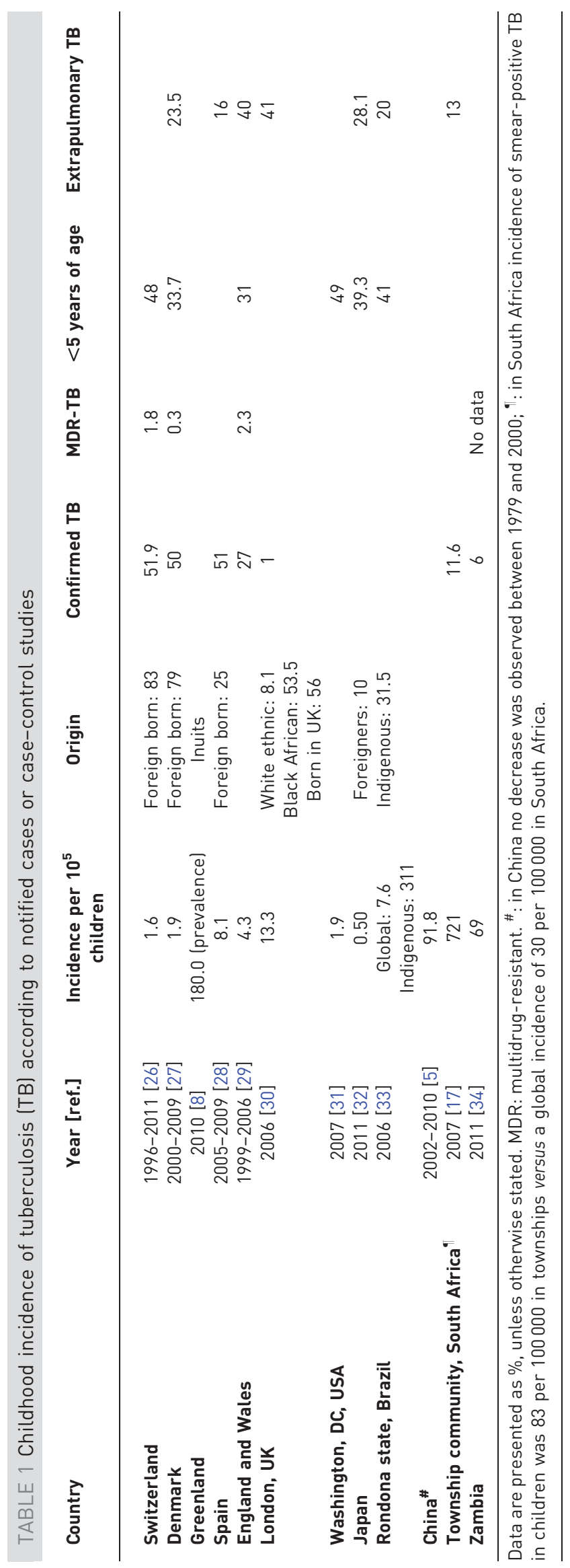




\section{Genetics}

As discussed previously, IFN- $\gamma$ and IL-12 are essential to defence against mycobacteria. Mendelian susceptibility to mycobacterial disease (MSMD) is a clinical syndrome that predisposes to infections caused by weakly virulent mycobacteria, such as BCG and by usual MTB strains [44]. Severe TB in conjunction with MSMD had been estimated to be present in between $5 \%$ and $40 \%$ of cases [44]. Several mutations in autosomal genes and genes within the X chromosome have been identified in MSMD, all of which affect the IL-12/IFN- $\gamma$ pathway [43-47]. Consequently, IFN- $\gamma$ has been reported to be partially effective for mycobacterial infection in IL-12 receptor $\beta 1$-deficient patients $[45,48]$. Genetic background also plays a role in tuberculin skin test (TST) responsiveness after BCG vaccination [49, 50].

\section{Diagnosis}

Symptoms of childhood TB are nonspecific, and up to $50 \%$ of children may be asymptomatic in the early stage [51]. The added burden of malnutrition and HIV further confound the diagnosis of childhood TB. To improve consistency among authors, experts have recently proposed clinical case definition categories (confirmed, possible, probable, unlikely, or not TB) for intrathoracic disease in symptomatic children [52].

\section{Immunological tests}

Immunological tests demonstrating T-cell reactivity to MTB derivatives are fundamental to LTBI diagnosis [53]. TST is still considered the gold standard compared with IFN- $\gamma$ release assays (IGRAs). Neither IGRA nor TST are designed to diagnose TB, as they do not distinguish between LTBI and TB disease [35, 54].

BCG vaccination may interfere with TST results in low-TB prevalence communities, while in high-TB burden populations its impact on positivity is limited to young children $<4$ years of age $[35,49]$. Reduced levels of TST positivity, as low as 20\%, have been reported in children with TB disease [18]. Furthermore, fewer extrapulmonary TB patients than pulmonary TB patients had positive TST results [5]. However, a cohort study showed a predictive interest for TST value in children aged 6-10 years, as a strong tuberculin reaction predicted TB in adolescents after an initial quiescent period [22].

In LTBI, good agreement between IGRA tests was reported, while agreement between TST and IGRA was moderate, particularly in older children $[8,54,55]$. Within contacts, the proportion of children with positive IGRA increased with adult infectiveness [56]. IGRA responses were similar regardless of contacts BCG status [56]. In the absence of a gold standard for LTBI, the "true" sensitivity of these tests cannot be assessed directly and the predominately sensitivity driven negative predictive value for progression in the first years, following recent infection, is very high and nearly identical for both IGRAs [57]. According to a meta-analysis, during active TB, IGRA sensitivity range in children was $60-100 \%$ and specificity was $85 \%$ [58]. IGRAs could be more useful in TB disease when using cells from the site of disease, for example bronchoalveolar lavage or cerebrospinal fluid (CSF) $[53,59]$.

Rates of indeterminate IGRA results vary from 0 to $35 \%$, and are particularly high in very young children and immunocompromised populations [53-56, 59]. HIV infection decreased positive IGRA rate, even in the presence of a well-preserved CD4 count [60]. In refugee children, IGRA failure rates reached 15\%, largely because of insufficient mononuclear leukocyte yields [61]. Consequently, recommended cut-off points were questioned in young children [37].

Recent studies have identified markers measured in Quantiferon (Cellestis Limited, Carnegie, Australia) supernatants, potentially able to discriminate between LTBI and TB disease, such as IFN- $\alpha 2$, IL-1 receptor- $\alpha$, sCD40L, vascular endothelial growth factor and IP-10, that are even expressed in cases of HIV infection $[56,62]$. Using TST, IGRA and IP-10 together, and considering any positive test, would identify LTBI in $70 \%$ of contacts exposed to paucibacillary adult cases. These data suggest that a significant proportion of children with asymptomatic infections may be missed by a single test [56].

A gold standard for LTBI diagnosis in children is still lacking. In low- and middle-income countries, IGRA use is not recommended in children irrespective of HIV status [63]. In high-income countries, recommendations vary as to whether IGRA should replace TST or be used as a confirmatory tool.

\section{Bacteriological confirmation}

The absence of an operational gold standard to diagnose childhood TB has encouraged the use of latent class analysis [64]. However, the resulting sensitivity and specificity are highly dependent on the previously assumed relationships and clinical scoring is no longer recommended [63]. Indeed, bacteriological confirmation is mandatory whenever possible [63]. Smear microscopy performs poorly in both sputum and gastric liquid aspirates (GLA). Among TB suspects, the culture yield for MTB per patient is $<15 \%[5,65]$. As TB disease is 
TABLE 2 New sampling methods and laboratory techniques

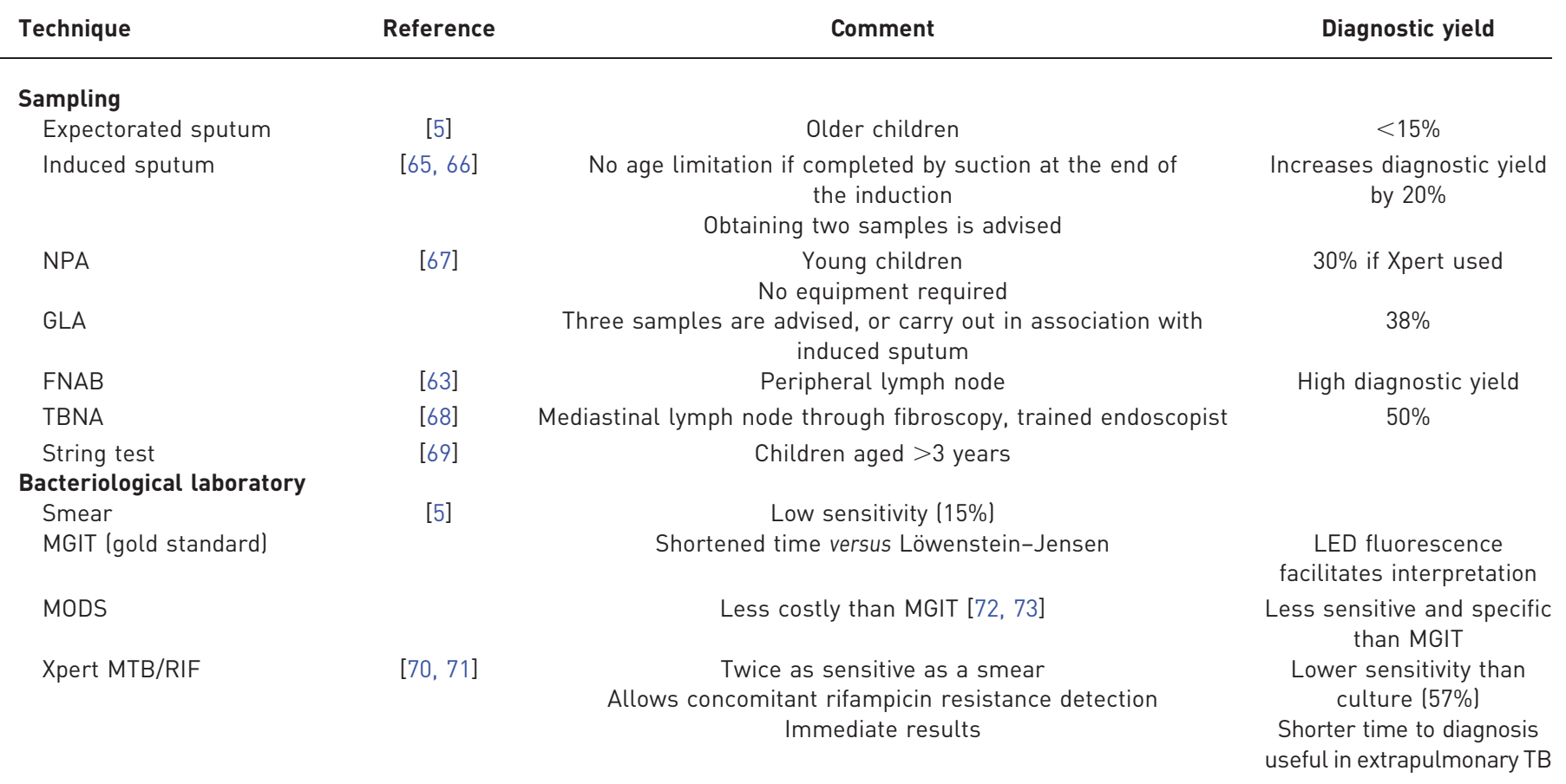

NPA: nasopharyngeal aspirates; GLA: gastric liquid aspirates; FNAB: fine-needle aspiration biopsy; TBNA: transbronchial needle aspiration; MGIT: mycobacterial growth indicator tube; MODS: microscopic observation drug susceptibility; TB: tuberculosis.

confirmed bacteriologically much more frequently in prospective studies than in observational data, new sampling and laboratory techniques should dramatically improve the diagnosis (table 2).

\section{Sample collection}

Alternative methods of sample collection include nasopharyngeal aspiration (NPA), hypertonic salineinduced sputum collection, which is reported to be more sensitive than GLA [24, 65], string test and lymph node fine-needle aspiration biopsy (FNAB). Induced sputum was reported to be successful in the majority of the patients, irrespective of age, without any major adverse event increasing the diagnostic yield by $>20 \%$ $[65,66]$. Although less frequently positive than induced sputum, NPA is easy to perform [67]. String test has been proposed as an adjunctive sample tool, and is well tolerated by patients as young as 4 years of age [69]. Data are still lacking on its efficiency in TB diagnosis. FNAB of enlarged peripheral lymph glands has been demonstrated to give a high bacteriological yield $[63,69]$. In high-risk populations, FNAB using a combination of cytomorphology, autofluorescence, and Ziehl-Nielsen staining provides a rapid and definitive diagnosis of mycobacterial infection in $60 \%$ of the children sampled [69].

\section{New bacteriological techniques}

The microscopic observation drug susceptibility (MODS) assay is an inexpensive liquid culture-based method. In children with TB, MODS demonstrated a higher sensitivity than smear. A MODS assay required a shorter median time to detect MBT growth than usual liquid culture, but was less sensitive and specific [72, 73].

The Xpert MTB/RIF assay (Cepheid Inc., Sunnyvale, CA, USA) is the most popular molecular diagnosis test. Xpert MTB/RIF represents a paradigm shift in the diagnosis of TB and drug-resistant TB by simultaneously detecting MTB and rifampicin resistance-conferring mutations in a closed system suitable for use outside conventional laboratory settings in less than $2 \mathrm{~h}$, directly from sputum samples [74]. In children with pulmonary TB, Xpert MTB/RIF consistently outperformed sputum and GLA smear tests, diagnosing $47 \%$ of smear-negative TB patients $[6,51,70,75]$. For smear-negative cases, the incremental increase in sensitivity from testing a second specimen was $25 \%$ for Xpert MTB/RIF, compared with $13-17 \%$ for culture [67]. Using Xpert MTB/RIF on two induced sputum specimens detected twice as many cases (75.9\%) compared with sputum smear (38\%) resulting in an overall Xpert MTB/RIF specificity of $98.8 \%$. Xpert MTB/RIF 
diagnosed pulmonary TB in 51.2\% of older children in a high HIV prevalence setting [74]. However, several specimens produced an indeterminate Xpert MTB/RIF result and had to be repeated [70].

Compared with culture, the pooled sensitivity estimate of Xpert MTB/RIF on sputum and GLA was $96 \%$ in smear-positive and $55 \%$ in smear-negative children, with a pooled specificity of $\geqslant 98 \%$ for all groups $[70,71,74,76]$. Xpert MTB/RIF showed a trend towards reduced sensitivity among children aged 0-4 years or with HIV infection $[6,76]$. High sensitivity (87\%) of Xpert MTB/RIF was reported in samples from children with extrapulmonary TB [76, 77]. Specificity values varied from $81 \%$ to $99 \%$ [76, 77]. Sensitivity was increased in pleural fluid (100\%) and peripheral lymph nodes (86\%) compared with CSF (75\%) [77].

Among smear-positive, culture-negative cases, some were Xpert MTB/RIF positive and might represent false negatives [70], questioning whether culture can be considered the "gold standard" in children. Indeed, an improved diagnostic technique should have low specificity relative to culture, identifying disease missed by culture [78].

The most recent WHO recommendations [63] are that Xpert MTB/RIF should be used rather than conventional microscopy and culture as the initial diagnostic test in children suspected of having multidrug-resistant (MDR)-TB or HIV-associated TB, or in cases of TB meningitis testing CSF [63, 74].

A genome-wide RNA expression study undertaken in a large population of children with suspected TB identified a 51-transcript signature distinguishing TB from other diseases with a sensitivity of $82 \%$ and a specificity of $83 \%$ against culture [71]. The risk score also distinguished TB disease from LTBI, with a sensitivity of $94 \%$ and a specificity of $100 \%$ [71]. A 10-gene signature correctly classified $78 \%$ of TB cases in Amerindian children [79]. However, the translation of transcriptional signatures into diagnostic tools is still challenging.

Urinary detection of lipoarabinomannan is considered useful in HIV-positive adults, but this test showed insufficient sensitivity and specificity in children [80]. The electronic nose, using either urine and/or breath volatile organic compound biomarkers, displayed low sensitivity and specificity [81].

Multiple samples from different sites and expanding Xpert MTB/RIF use could dramatically improve confirmation rates in childhood TB. However, simple and sensitive tests, which are easy to perform in infants and young children, are still required.

\section{Drug-resistant TB}

Among children living with drug-resistant patients, an overall high prevalence of TB was observed (7.5\%), regardless of the age of the child [82]. In places where the rates of drug-resistant TB have been monitored, the rates among children were similar or slightly higher than those among adults $[2,20]$. In Namibia, South Africa, Germany, the UK and the USA, MDR-TB was positively associated with being aged $<15$ years [20]. An Egyptian study detected an overall drug resistance of $24 \%$ in children with TB, with a MDR-TB prevalence of $2.7 \%$. Alarmingly, resistance to second-line drugs was $2.7 \%$ for amikacin and $1.4 \%$ for ciprofloxacin [83]. In Thailand, 5.7\% of specimens from children were MDR-TB in 2012 revealing a drastic increase in a few years [51]. Furthermore, in a recent Indian survey, half of the children with drug sensitivity tests (DSTs) harboured drug-resistant strains, of which 50\% were MDR-TB [18]. An epidemic of extensively drug-resistant (XDR)-TB was discovered in an Italian school via contact tracing of a 12-year-old patient (Italian native) with pulmonary XDR-TB. Five cases were diagnosed with TB and 19 with LTBI (12 out of 19 were in children). The index case was probably a schoolmate originating from Eastern Europe [84].

Diagnosing drug resistance requires culture and DST. Consequently, initiation of appropriate treatment has been delayed in children for between 2 and 9 months [85]. Xpert MTB/RIF has improved the diagnosis of drug resistance giving immediate results. It was $86-100 \%$ sensitive and $97 \%$ specific for the detection of rifampicin resistance in children compared with culture. However, when possible, confirmation with classical DST is still required [20, 51, 70, 76]. More work should be done to improve Xpert MTB/RIF sensitivity in paucibacillary patients $[20,51]$.

While adults represented the contamination source in most cases, in resource-limited settings with a highburden of TB, the results of DST from adult index cases were not systematically used to investigate drug resistance in children [20]. Moreover, the index case was not found in $17-45 \%$ of cases [86, 87], highlighting the importance of improved reverse contact tracing. However, in TB high-burden countries, children may be exposed to several TB cases, with different susceptibility patterns [88].

Few children had a previous diagnosis of $\mathrm{TB}$, suggesting that most children acquired an already resistant strain from their index case $[87,88]$. However, in the most recent South African survey, 59\% of children who were infected with HIV had previously received $>1$ month of anti-TB treatment, compared with $15 \%$ for children who were not HIV infected. Drug resistance occurred in a significantly higher proportion of children who had received previous treatment for TB than in untreated patients [88]. In Thailand, prior treatment for $\mathrm{TB}$ disease was associated with drug resistance, while this was not the case with anterior 
treatment for LTBI [51]. Children living in settings where the prevalence of HIV is high or where resistance to isoniazid is high should be suspected of MDR-TB [89].

\section{Imaging}

Patients $<3-4$ years of age have the highest lymph node involvement, but the least parenchymal lesions compared with older children $[52,90]$. The pivotal role of chest radiography in TB diagnosis is, however, impaired in young children, without any improvement when adding a lateral view [91]. In a South African study of TB suspects, although $75 \%$ of culture-positive children were symptomatic, only $28 \%$ demonstrated radiological evidence suggestive of TB [92].

While computed tomography (CT) is more sensitive than chest radiography [9, 91, 93], attitudes towards children with lesions observed only on CT scans are contrasting. Some authors consider that these abnormalities should be ignored and the case classified as LTBI [19]. During an outbreak of TB in a nursery, based on CT scans, $96 \%$ of the TST-positive children were diagnosed with TB disease [9]. In $60-85 \%$ of children with normal chest radiography, the CT scan showed adenopathies or a pathological infiltrate $[9,90]$. In infants, chest CT scans revealed extensive parenchymal lesions and hilar/mediastinal lymph node enlargement in all cases [94].

Airway complications are frequently missed by chest radiography. Compared with endoscopy, CT scan sensitivity for prediction of severe bronchial involvement was $100 \%$, but specificity was only $72 \%$ [95].

\section{Bronchoscopy}

Early detection and effective treatment of endobronchial TB is essential in order to decrease secondary bronchial stenosis and bronchiectasis [95-97]. Fibroscopy showed airway anomalies in half of the children with lymphadenopathy on chest radiography. Severe disease with gross extrinsic compression or obstructive endoluminal mass was found in $18.9 \%$ of the cases. However, several patients with severe disease were symptom free [95]. Mediastinal lymph nodes may be sampled safely using transbronchial needle aspiration (TBNA) through flexible bronchoscopy [68]. A prospective study in 28 children with large subcarinal lymphadenopathy reported that TBNA provided a definitive diagnosis of mycobacterial infection in $50 \%$ of patients [68].

CT scan and bronchoscopy are useful in infants and young children whenever usual tools are inconclusive. They are of particular interest when bronchial complications are suspected.

\section{HIV co-infection}

In 2009, an estimated 2.5 million children $<15$ years of age were infected with HIV, with the majority living in sub-Saharan Africa [98]. HIV prevalence among children with TB, in countries with moderate-to-high prevalence, ranges from $10 \%$ to $60 \%$ [99]. In the UK and USA, the incidence of TB in HIV-infected children was less than 1 per 100 child-years contrasting dramatically with the value of 23 per 100 child-years in South African townships [99]. Furthermore, according to South African surveys, HIV co-infection increased in 10 years from $7 \%$ to $26 \%$ of the tested children with TB [88]. Advanced immunosuppression and receiving antiretroviral therapy (ART) for $<6$ months drastically increase the risk of TB [98]. In the absence of ART, more than $20 \%$ of HIV-infected children living in high-burden areas would develop TB disease, leading to a three- to six-fold higher mortality $[98,100]$.

The diagnosis of TB disease in HIV-positive children is particularly difficult because of HIV-related comorbidities and chronic lung disease [24]. HIV-positive children must, therefore, be screened routinely for TB and HIV testing should be offered to all TB suspects [63]. Although HIV-positive children are more prone to develop disseminated and rapidly progressive TB, the clinical presentation is usually similar to that seen in HIV-uninfected children, but with more severe features such as concomitant extrapulmonary TB [24, 101]. Cavitation is infrequent [101]. Sophisticated diagnostic techniques are more frequently required in HIV patients.

Immune reconstitution syndrome (IRIS) is observed after the initiation of ART in severely immunocompromised patients. It induces transient worsening of TB manifestations such as fever, lymphadenopathy, pleural effusions, respiratory distress and exacerbation of cerebral tuberculomas [24]. In Thailand, IRIS was documented within 4 weeks of the initiation of ART in 19\% of children with low CD4 cell percentages [24]. However, extensive delays in starting ART should be avoided [99, 100]. Current recommendations for co-infected children are that ART should be initiated 2-8 weeks after starting TB treatment $[63,100,101]$. Early ART initiation is the most important intervention for reducing overall mortality and TB risk among HIV-infected infants $[2,102]$. Among children considered free of TB at ART enrolment, TB incidence was 6.28 per 100 child-years during days $0-90$ of ART, demonstrating the importance of initial TB screening [23]. A better integration of HIV and TB services with general child health clinics is required [103]. 


\section{Treatment}

Despite a recent WHO update on childhood TB treatment regimens (table 3), there is a lack of childfriendly formulations matching these new recommendations $[1,63]$.

The recommended isoniazid dose of $5 \mathrm{mg} \cdot \mathrm{kg}^{-1}$ body weight in childhood $\mathrm{TB}$ leads to lower serum concentrations than those recommended for adults [104]. Pharmacokinetics data confirmed that children aged $<2$ years achieve target concentrations using the revised WHO dosage recommendations [104, 105]. However, retrospectively, reported clinical responses to treatment and outcomes were similar with current recommended dosages and with lower dosages [106]. Intermittent treatment is less effective in achieving cure in children than daily doses, even if it is better tolerated in some cases [107, 108]. Addition of a fourth drug such as ethambutol is necessary in complicated pulmonary TB or meningeal involvement [36]. Safety of ethambutol in children is confirmed, but recommended doses are probably too low for young children $[24,109]$. For infants $<6$ months of age with widespread dissemination, ethionamide use was reported in place of ethambutol considering its superior penetration into the central nervous system [94].

Children aged $<1$ year and those with severe TB or extrapulmonary TB were more likely to have poor treatment outcomes [5]. Paradoxical unexplained deteriorations occurred in 14\% of non-HIV paediatric patients within a median time of 80 days. Many patients improved with corticosteroids, suggesting an exacerbated immunological response [110].

A poorer response to treatment among HIV-infected children leads to prolonging TB treatment to 9 months, although there is no evidence supporting this practice $[24,101]$. In addition, the effect of HIV co-infection on the pharmacokinetics of anti-TB drugs in children requires further investigation [104].

In high-prevalence low-resource settings, the concept of relevant disease is used to decide which children will be treated, and leads to consideration of mild TB cases as LTBI [24]. However, there is no evidence of the accuracy of this concept, which can be summarised as choosing between treating patients or limiting disease diffusion within the community $[1,24]$. In most settings, children with TB continue to be given low priority by national TB control programmes because they are less likely to transmit disease [1].

\section{Additional medications}

Steroid therapy is usually indicated in cases of severe bronchial obstruction [111]. Combining 4 weeks of steroids and possibly endoscopic resection demonstrated good results and dramatically reduced the need for surgery $[95,96]$. In severe pulmonary TB, additional antibiotics may be required for bacterial co-infections [94].

\section{Antiretroviral therapy}

The optimal antiretroviral regimen for children receiving TB treatment has not been established, as rifampicin induces significant reductions in serum levels of several ART drugs. Rifabutin has fewer drug interactions but is associated with ocular toxicity [63]. In older children receiving rifampicin, two nucleoside reverse transcriptase inhibitors should be associated with Efavirenz; additional studies are required for young children [112]. Treatment should not be interrupted during IRIS except in cases of central nervous system TB, where IRIS can have devastating consequences [2]. Severe IRIS may require a course of glucocorticoids [2].

New recommended regimens are widely used for childhood TB. However, specially designed randomised controlled trials determining the best drug associations and required treatment duration are still required in children.

\section{TABLE 3 Recommended daily dosages of anti-tuberculous drugs}

\begin{tabular}{lccc} 
Drug & Mean dose & Range & Maximal dose \\
\hline Isoniazid (H) $^{\#}$ & $10 \mathrm{mg} \cdot \mathrm{kg}^{-1}$ & $7-15 \mathrm{mg} \cdot \mathrm{kg}^{-1}$ & $300 \mathrm{mg} \cdot$ day $^{-1}$ \\
Rifampicin (R) & $15 \mathrm{mg} \cdot \mathrm{kg}^{-1}$ & $10-20 \mathrm{mg} \cdot \mathrm{kg}^{-1}$ & $600 \mathrm{mg} \cdot$ day $^{-1}$ \\
Pyrazinamide (Z) & $35 \mathrm{mg} \cdot \mathrm{kg}^{-1}$ & $30-40 \mathrm{mg} \cdot \mathrm{kg}^{-1}$ & \\
Ethambutol (E) & $20 \mathrm{mg} \cdot \mathrm{kg}^{-1}$ & $15-25 \mathrm{mg} \cdot \mathrm{kg}^{-1}$ & \\
Amikacin & & $15-22.5 \mathrm{mg} \cdot \mathrm{kg}^{-1}$ & $1000 \mathrm{mg}$ \\
Ofloxacin & & $15-20 \mathrm{mg} \cdot \mathrm{kg}^{-1} \mathrm{twice}$ daily & $800 \mathrm{mg}$ \\
Ethionamide & $15-20 \mathrm{mg} \cdot \mathrm{kg}^{-1}$ twice daily & $1000 \mathrm{mg}$ \\
Cycloserine & $10-20 \mathrm{mg} \cdot \mathrm{kg}^{-1}$ once/twice daily & $1000 \mathrm{mg}$ \\
Para-aminosalicylic acid & & $150 \mathrm{mg} \cdot \mathrm{kg}^{-1} 2-3$ times daily & $12000 \mathrm{mg}$
\end{tabular}

\#: the higher end of the range for isoniazid dose applies to younger children, as the children grow older the lower end of the dosing range becomes more appropriate. Data from [63]. 


\section{Second-line drugs for MDR-TB}

Despite advanced disease at presentation and a high prevalence of HIV co-infection, children with MDR-TB can be treated successfully, using individualised treatment under routine conditions [86]. A meta-analysis reported excellent outcomes in children, with a cure rate of $82 \%$, mortality of $6 \%$ and adverse events in $39 \%$ of cases [85]. Mortality was associated with malnutrition, HIV and extrapulmonary TB [86]. Lack of weight gain was a warning sign for treatment failure [85, 105].

The South African protocol included, in the majority of cases, high-dose isoniazid as isolates with an inhA promoter region mutation usually have a low minimum inhibitory concentration [105]. Benefits of fluoroquinolones outweighed the risks and they should be use systematically $[63,86]$. Ethionamide, paraaminosalycilic acid and cycloserine were used successfully, but tolerance was controversial $[36,86,87,113]$. Success was higher when treatment included injectable drugs, although these induced hearing deficit [36]. Linezolid-containing regimens can be effective even after failing second-line treatment, but adverse events should be monitored [114].

In the absence of a sufficient number of medicines to which a strain is sensitive in vivo, life-saving treatment may rely on the use of new medicines such as bedaquiline or delamanid, despite the absence of data in children. The European Respiratory Society/WHO TB Consilium was implemented to face drug-resistant $\mathrm{TB}$ in Europe. It supports clinicians through a platform that rapidly provides four expert opinions on how best to manage a complex case [84]. It recently recommended inclusion of one of either bedaquiline or delamanid in a regimen that included meropenem and clofazimine for a 12-year-old patient with XDR-TB. Delamanid was procured in a compassionate way via the manufacturer. Initial clinical, laboratory and radiological improvements were observed [84].

Reported results of MDR-TB treatment are encouraging, but well conducted clinical trials are urgently needed for MDR-TB in children, to improve treatment safety and efficiency.

\section{Prevention}

$B C G$

BCG remains the only available vaccine for TB worldwide [35]. The global BCG efcacy is estimated to be $\sim 50 \%$, ranging from a zero effect to $80 \%[115,116]$. In addition, BCG vaccination at birth halved neonatal mortality from non-TB infections [117]. At US \$2-3 per dose, BCG vaccination costs US \$206 per year of healthy life gained [118].

As BCG is a live vaccine, the absence of a competent immune response can lead to disseminated BCG disease in $1 \%$ of $\mathrm{HIV}$-infected infants with an all-cause mortality rate of $75-86 \%$. IRIS frequency after vaccination is estimated to be $\sim 15 \%$ in HIV-infected children [119]. Consequently, the WHO Global Advisory Committee on Vaccine Safety recommends that BGC should not be administered in HIV-infected patients [1]. However, a case-control study reported that childhood BCG that caused a scar decreased TB risk in adulthood by $70 \%$, irrespective of HIV status $[6,115]$. In case of disseminated BCG disease, the appropriate combination of anti-TB drugs should be used taking into account inherent resistance to pyrazinamide and variable BCG resistance to isoniazid [119].

BCG discontinuation demonstrated adverse effects in low incidence countries, for example France or Sweden. Universal BCG vaccination replacement by a targeted vaccination programme resulted in very low BCG vaccination coverage, leading to a 15 -fold increase in TB incidence in children born to parents of foreign origin [120, 121].

The dynamic interaction of age and immunity, as well as its influence on pathogen evolution, needs to be considered in the development of future vaccination strategies. The best time for vaccination should be determined as maximal efficacy is not obtained before the age of 2 years, while BCG realised at birth prevents severe diseases in infants [21]. The modern vaccine should also protect against milder diseases [92]. The development pipeline now includes 12 new vaccines which can either boost the initial effects of BCG or replace BCG [122]. However, no new vaccine has shown effectiveness in late phase trials [122].

\section{Isoniazid preventive therapy}

While TB control is aimed at reducing the incidence of TB by early diagnosis and treatment of infectious cases of TB, TB elimination requires focus on sterilising the pool of latently infected individuals, from whom future TB cases would be generated [57].

Isoniazid prophylaxis reduces the risk of developing TB by $59 \%$ among children aged $\leqslant 15$ years, excluding children in whom primary prophylaxis was initiated before 4 months of age $[4,35,123]$. It was more effective among children aged 5-15 years than among children aged $<5$ years [123]. Screening for disease 
and treatment with isoniazid preventive therapy (IPT), without testing for MTB infection, is considered the most cost-effective strategy in 0-2-year-old children [124].

A marked protective effect was demonstrated against TB disease and mortality among HIV-infected children [125], so IPT is recommended for any HIV-positive child with TB exposure, irrespective of the child's age or TST result, once active TB has been excluded [24]. However, there is, as yet, not enough data to guide the duration of prophylaxis, or to support its use in children on ART and in those living in low TB prevalence areas, without TB contact $[126,127]$. The protection offered by 6 months IPT appears to be short-lasting among HIV-infected persons in high-burden TB areas [128]. In the same way, IPT was safe but ineffective as pre-exposure prophylaxis against $\mathrm{TB}[2,129]$.

A low peak serum concentration of isoniazid was found among young children given isoniazid daily at 4-6 mg $\mathrm{kg}^{-1}$, and the recommended dosage is $10 \mathrm{mg} \cdot \mathrm{kg}^{-1}$ [13]. However, a daily dosage of $5 \mathrm{mg} \cdot \mathrm{kg}^{-1}$ among young children was used in virtually all clinical trials that investigated IPT efficacy, questioning the necessity of using a higher dosage [128]. Providing IPT to young children poses a small risk of isoniazid monoresistant TB while in adolescents IPT poses a considerably higher risk [24, 130].

Field effectiveness may be substantially lower as a result of nonadherence that approximates $76 \%$ in South Africa $[63,123,128]$. A short-course regimen with isoniazid and rifampicin for 3 months was superior to a 9 month course of isoniazid monotherapy regarding compliance and chest radiography evolution, and showed a significant decrease in childhood TB that persisted to 12 years of follow-up [109, 131]. Data are limited for rifampicin and pyrazinamide combination therapy and for economic assessment of any regimens in children [132]. In children aged $>12$ years, high dose isoniazid and rifapentine combination therapy once a week for 3 months demonstrated high completion rates despite frequent adverse events [109, 133].

IPT is under prescribed and monitored. Less than $20 \%$ of the European countries collect information on LTBI treatment outcomes [134]. Among South African children hospitalised for TB, IPT missed opportunities occurred in $64 \%$ [123].

Pragmatic solutions are urgently required. Parents are often reluctant to provide preventive treatment for an otherwise well child, and are further discouraged by the long duration of preventive therapy [2].

There is evidence to support 6 months of rifampicin for contacts of isoniazid-resistant disease and it is recommended for this category in the UK [109]. MDR-TB prophylaxis is much more controversial. The high failure rate of isoniazid or isoniazid and rifampicin chemoprophylaxis and first-line therapy in children with known household exposure to MDR-TB enhances the urgent need for the evaluation of appropriate chemoprophylactic regimens for child contacts of patients with MDR-TB [88]. The WHO recommends that symptomatic children who are TST positive with a MDR-TB contact, with normal chest radiography and negative bacteriological results, should be treated with a broad-spectrum antibiotic inactive on MTB and closely monitored. If the clinical condition gradually deteriorates, empirical treatment of MDR-TB should be established [89].

A completely different approach would be to prevent the reactivation of LTBI by post-exposure vaccination, which is one of the targets of the ongoing research into development of new TB vaccines [57].

\section{Conclusion}

Towards zero death, the WHO roadmap fixes as long-term objectives for 2025 the possibility of prediction of disease progression in infected children, and implementation of new efficient vaccines against infection and disease. The roadmap insists on the necessity of shorter child-friendly treatment regimens, which have been accurately designed and tested. As an immediate point of action it underlines the need to focus on TB and HIV in pregnant females and young mothers as the best way to protect the child [3]. However, to achieve the goal of TB elimination by 2050 , it will be necessary to combine the transmission-blocking approach with wellresourced LTBI treatment strategies, accelerating towards zero infections, zero cases and zero deaths [57, 135].

\section{Acknowledgements}

We would like to thank Deepa Rajan (World Health Organization, Geneva, Switzerland) who reviewed the manuscript.

\section{References}

1 Sandgren A, Cuevas LE, Dara M, et al. Childhood tuberculosis: progress requires an advocacy strategy now. Eur Respir J 2012; 40: 294-297.

Perez-Velez CM, Marais BJ. Tuberculosis in children. N Engl J Med 2012; 367: 348-361.

World Health Organization. Roadmap for Childhood Tuberculosis: Towards Zero Deaths. Geneva, WHO, 2013

Gomes VF, Andersen A, Wejse C, et al. Impact of tuberculosis exposure at home on mortality in children under 5 years of age in Guinea-Bissau. Thorax 2011; 66: 163-167. 

130: e1433-e1440.

Zar HJ, Udwadia ZF. Advances in tuberculosis 2011-2012. Thorax 2013; 68: 283-287.

7 Teo SS, Alfaham M, Evans MR, et al. An evaluation of the completeness of reporting of childhood tuberculosis. Eur Respir J 2009; 34: 176-179.

8 Soborg B, Koch A, Thomsen VØ, et al. Ongoing tuberculosis transmission to children in Greenland. Eur Respir J 2010; 36: 878-884.

9 Garrido JB, Alías Hernández I, Bonillo Perales A, et al. Usefulness of thoracic CT to diagnose tuberculosis disease in patients younger than 4 years of age. Pediatr Pulmonol 2012; 47: 895-902.

10 Pang J, Teeter LD, Katz DJ, et al. Epidemiology of tuberculosis in young children in the United States. Pediatrics 2014; 133: e494-e504.

11 Baghaie N, Khalilzadeh S, Bolursaz MR, et al. Contact tracing of a 15-year-old girl with smear-negative pulmonary tuberculosis in Tehran. East Mediterr Health J 2012; 18: 399-401.

12 Verver S, van Loenhout-Rooyackers JH, Bwire R, et al. Tuberculosis infection in children who are contacts of immigrant tuberculosis patients. Eur Respir J 2005; 26: 126-132.

13 World Health Organization. Recommendations for Investigating Contacts of Persons with Infectious Tuberculosis in Low- and Middle-Income Countries. Geneva, WHO, 2012.

14 Tagarro A, Jiménez S, Sánchez A, et al. Brote de tuberculosis en un colegio de primaria: descripcion y consideraciones sobre el valor del jugo gastrico en el manejo de microepidemias [Tuberculosis outbreak in a primary school: description and reflections on the value of gastric juice in the management of micro-epidemics]. Enferm Infecc Microbiol Clin 2011; 29: 90-95.

15 Døllner H, Ramm CT, Harstad I, et al. Risk of developing tuberculosis after brief exposure in Norwegian children: results of a contact investigation. BMJ Open 2012; 2: e001816.

16 Mandalakas AM, Kirchner HL, Lombard C, et al. Well-quantified tuberculosis exposure is a reliable surrogate measure of tuberculosis infection. Int J Tuberc Lung Dis 2012; 16: 1033-1039.

17 Middelkoop K, Bekker LG, Morrow C, et al. Childhood infection and disease: a spatial and temporal transmission analysis in a South African township. S Afr Med J 2009; 99: 738-743.

18 Jain SK, Ordonez A, Kinikar A, et al. Pediatric tuberculosis in young children in India: a prospective study. Biomed Res Int 2013; 2013: 783698.

19 Marais BJ, Parker SK, Verver S, et al. Primary and postprimary or reactivation tuberculosis: time to revise confusing terminology? AJR Am J Roentgenol 2009; 192: W198.

20 Zignol M, Sismanidis C, Falzon D, et al. Multidrug-resistant tuberculosis in children: evidence from global surveillance. Eur Respir J 2013; 42: 701-707.

21 Donald PR, Marais BJ, Barry CE 3rd. Age and the epidemiology and pathogenesis of tuberculosis. Lancet 2010; 375 : 1852-1854.

22 Leung CC, Yew WW, Au KF, et al. A strong tuberculin reaction in primary school children predicts tuberculosis in adolescence. Pediatr Infect Dis J 2012; 31: 150-153.

23 Dangor Z, Izu A, Moore DP, et al. Temporal association in hospitalizations for tuberculosis, invasive pneumococcal disease and influenza virus illness in South African children. PLoS One 2014; 9: e91464.

24 Marais BJ, Graham SM, Cotton MF, et al. Diagnostic and management challenges for childhood tuberculosis in the era of HIV. J Infect Dis 2007; 196: Suppl. 1, S76-S85.

25 Auld AF, Tuho MZ, Ekra KA, et al. Tuberculosis in human immunodeficiency virus-infected children starting antiretroviral therapy in Côte d'Ivoire. Int J Tuberc Lung Dis 2014; 18: 381-387.

26 Oesch Nemeth G, Nemeth J, Altpeter E, et al. Epidemiology of childhood tuberculosis in Switzerland between 1996 and 2011. Eur J Pediatr 2014; 173: 457-462.

27 Hatleberg CI, Prahl JB, Rasmussen JN, et al. A review of paediatric tuberculosis in Denmark: 10-year trend, 2000-2009. Eur Respir J 2014; 43: 863-871.

28 Rodríguez Valín E, Garrido Estepa M, Villarrubia Enseñat S, et al. Epidemiologia de la tuberculosis pediatrica en Espana, 2005-2009 [Epidemiology of childhood tuberculosis in Spain: 2005-2009]. Rev Esp Salud Publica 2012; 86: 49-59.

29 Abubakar I, Laundy MT, French CE, et al. Epidemiology and treatment outcome of childhood tuberculosis in England and Wales: 1999-2006. Arch Dis Child 2008; 93: 1017-1021.

30 Ruwende JE, Sanchez-Padilla E, Maguire H, et al. Recent trends in tuberculosis in children in London. J Public Health (Oxf) 2011; 33: 175-181.

31 Siegel D, Song X, Klontz K, et al. Epidemiology of childhood tuberculosis: use and evaluation of the pediatric health information system to assess local and national incidence. Pediatr Infect Dis J 2011; 30: 428-430.

32 Tuberculosis Surveillance Center, RIT, JATA. [Tuberculosis Annual Report 2011- (2) Childhood and elderly tuberculosis]. Kekkaku 2013; 88: 611-616.

33 Gava C, Malacarne J, Rios DP, et al. Tuberculosis in indigenous children in the Brazilian Amazon. Rev Saude Publica 2013; 47: 77-85.

34 Kapata N, Chanda-Kapata P, O'Grady J, et al. Trends in childhood tuberculosis in Zambia: a situation analysis. J Trop Pediatr 2013; 59: 134-139.

Gideon HP, Flynn JL. Latent tuberculosis: what the host "sees"? Immunol Res 2011; 50: 202-212.

Piccini P, Chiappini E, Tortoli E, et al. Clinical peculiarities of tuberculosis. BMC Infect Dis 2014; 14: Suppl. 1, S4. Erkens CG, Kamphorst M, Abubakar I, et al. Tuberculosis contact investigation in low prevalence countries: a European consensus. Eur Respir J 2010; 36: 925-949.

38 Ford CB, Lin PL, Chase MR, et al. Use of whole genome sequencing to estimate the mutation rate of Mycobacterium tuberculosis during latent infection. Nat Genet 2011; 43: 482-486.

39 Dheda K, Schwander SK, Zhu B, et al. The immunology of tuberculosis: from bench to bedside. Respirology 2010; 15: 433-450.

40 Walzl G, Ronacher K, Hanekom W, et al. Immunological biomarkers of tuberculosis. Nat Rev Immunol 2011; 11: 343-354.

41 Ottenhoff THM. The knowns and unknowns of the immunopathogenesis of tuberculosis. Int J Tuberc Lung Dis 2012; 16: 1424-1432. 
42 Gourgouillon N, de Lauzanne A, Cottart $\mathrm{CH}$, et al. TNF- $\alpha / \mathrm{IL}-2$ ratio discriminates latent from active tuberculosis in immunocompetent children: a pilot study. Pediatr Res 2012; 72: 370-374.

43 Kagina BM, Abel B, Scriba TJ, et al. Specific T cell frequency and cytokine expression profile do not correlate with protection against tuberculosis after bacillus Calmette-Guérin vaccination of newborns. Am J Respir Crit Care Med 2010; 182: 1073-1079.

44 de Beaucoudrey L, Samarina A, Bustamante J, et al. Revisiting human IL-12Rß1 deficiency: a survey of 141 patients from 30 countries. Medicine (Baltimore) 2010; 89: 381-402.

45 Boisson-Dupuis S, El Baghdadi J, Parvaneh N, et al. IL-12R $\beta 1$ deficiency in two of fifty children with severe tuberculosis from Iran, Morocco, and Turkey. PLoS One 2011; 6: e18524.

46 Mosaad YM, Soliman OE, Tawhid ZE, et al. Interferon- $\gamma+874$ T/A and interleukin-10 -1082 A/G single nucleotide polymorphism in Egyptian children with tuberculosis. Scand J Immunol 2010; 72: 358-364.

47 Sun L, Jin YQ, Shen C, et al. Genetic contribution of CISH promoter polymorphisms to susceptibility to tuberculosis in Chinese children. PLoS One 2014; 9: e92020.

48 Alangari AA, Al-Zamil F, Al-Mazrou A, et al. Treatment of disseminated mycobacterial infection with high-dose IFN- $\gamma$ in a patient with IL-12R $\beta 1$ deficiency. Clin Dev Immunol 2011; 2011: 691956.

49 Maes M, Verhagen LM, Ortega D, et al. Influence of Bacille Calmette-Guérin on tuberculin skin testing in Venezuelan Amerindians in high tuberculosis burden areas. J Infect Dev Ctries 2014; 8: 176-183.

50 Cobat A, Hoal EG, Gallant CJ, et al. Identification of a major locus, TNF1, that controls BCG-triggered tumor necrosis factor production by leukocytes in an area hyperendemic for tuberculosis. Clin Infect Dis 2013; 57: 963-970.

51 Lapphra K, Sutthipong C, Foongladda S, et al. Drug-resistant tuberculosis in children in Thailand. Int J Tuberc Lung Dis 2013; 17: 1279-1284.

52 Graham SM, Ahmed T, Amanullah F, et al. Evaluation of tuberculosis diagnostics in children: 1. Proposed clinical case definitions for classification of intrathoracic tuberculosis disease. Consensus from an expert panel. J Infect Dis 2012; 205: Suppl. 2, S199-S208.

53 Mazurek GH, Jereb J, Vernon A, et al. Updated guidelines for using interferon $\gamma$ release assays to detect Mycobacterium tuberculosis infection - United States, 2010. MMWR Recomm Rep 2010; 59: 1-25.

54 Kampmann B, Whittaker E, Williams A, et al. Interferon- $\gamma$ release assays do not identify more children with active tuberculosis than the tuberculin skin test. Eur Respir J 2009; 33: 1374-1382.

55 Haustein T, Ridout DA, Hartley JC, et al. The likelihood of an indeterminate test result from a whole-blood interferon- $\gamma$ release assay for the diagnosis of Mycobacterium tuberculosis infection in children correlates with age and immune status. Pediatr Infect Dis J 2009; 28: 669-673.

56 Yassin MA, Petrucci R, Garie KT, et al. Use of tuberculin skin test, IFN- $\gamma$ release assays and IFN- $\gamma$-induced protein10 to identify children with TB infection. Eur Respir J 2013; 41: 644-648.

57 Diel R, Loddenkemper R, Zellweger JP, et al. Old ideas to innovate tuberculosis control: preventive treatment to achieve elimination. Eur Respir J 2013; 42: 785-801.

58 Sester M, Sotgiu G, Lange C, et al. Interferon- $\gamma$ release assays for the diagnosis of active tuberculosis: a systematic review and meta-analysis. Eur Respir J 2011; 37: 100-111.

59 Chiappini E, Bonsignori F, Accetta G, et al. Interferon- $\gamma$ release assays for the diagnosis of Mycobacterium tuberculosis infection in children: a literature review. Int J Immunopathol Pharmacol 2012; 25: 335-343.

60 Mandalakas AM, van Wyk S, Kirchner L, et al. Detecting tuberculosis infection in HIV-infected children: a study of diagnostic accuracy, confounding and interaction. Pediatr Infect Dis J 2013; 32: e111-e118.

61 Lucas M, Nicol P, McKinnon E, et al. A prospective large-scale study of methods for the detection of latent Mycobacterium tuberculosis infection in refugee children. Thorax 2010; 65: 442-448.

62 Chegou NN, Detjen AK, Thiart L, et al. Utility of host markers detected in Quantiferon supernatants for the diagnosis of tuberculosis in children in a high-burden setting. PLoS One 2013; 8: e64226.

63 World Health Organization. Guidance for National Tuberculosis Programmes on the Management of Tuberculosis in Children. 2nd Edn. Geneva, WHO, 2014.

64 Tuyisenge L, Ndimubanzi CP, Ndayisaba G, et al. Evaluation of latent class analysis and decision thresholds to guide the diagnosis of pediatric tuberculosis in a Rwandan reference hospital. Pediatr Infect Dis J 2010; 29: e11-e18.

65 Thomas TA, Heysell SK, Moodley P, et al. Intensified specimen collection to improve tuberculosis diagnosis in children from Rural South Africa, an observational study. BMC Infect Dis 2014; $14: 11$.

66 Moore HA, Apolles P, de Villiers PJ, et al. Sputum induction for microbiological diagnosis of childhood pulmonary tuberculosis in a community setting. Int J Tuberc Lung Dis 2011; 15: 1185-1190.

67 Zar HJ, Workman L, Isaacs W, et al. Rapid molecular diagnosis of pulmonary tuberculosis in children using nasopharyngeal specimens. Clin Infect Dis 2012; 55: 1088-1095.

68 Goussard P, Gie RP, Kling S, et al. The diagnostic value and safety of transbronchial needle aspiration biopsy in children with mediastinal lymphadenopathy. Pediatr Pulmonol 2010; 45: 1173-1179.

69 Achkar JM, Lawn SD, Moosa MY, et al. Adjunctive tests for diagnosis of tuberculosis: serology, ELISPOT for sitespecific lymphocytes, urinary lipoarabinomannan, string test, and fine needle aspiration. J Infect Dis 2011; 204: Suppl. 4, S1130-S1141.

70 Bates M, O'Grady J, Maeurer M, et al. Assessment of the Xpert MTB/RIF assay for diagnosis of tuberculosis with gastric lavage aspirates in children in sub-Saharan Africa: a prospective descriptive study. Lancet Infect Dis 2013; 13: $36-42$.

71 Anderson ST, Kaforou M, Brent AJ, et al. Diagnosis of childhood tuberculosis and host RNA expression in Africa. N Engl J Med 2014; 370: 1712-1723.

72 Ha DT, Lan NTN, Wolbers M, et al. Microscopic observation drug susceptibility assay (MODS) for early diagnosis of tuberculosis in children. PLoS One 2009; 4: e8341.

73 Nhu NT, Ha DT, Anh ND, et al. Evaluation of XpertMTB/RIF and MODS assay for the diagnosis of pediatric tuberculosis. BMC Infect Dis 2013; 13: 31.

74 Weyer K, Mirzayev F, Migliori GB, et al. Rapid molecular TB diagnosis: evidence, policy making and global implementation of Xpert MTB/RIF. Eur Respir J 2013; 42: 252-271.

75 Nicol MP, Workman L, Isaacs W, et al. Accuracy of the Xpert MTB/RIF test for the diagnosis of pulmonary tuberculosis in children admitted to hospital in Cape Town, South Africa: a descriptive study. Lancet Infect Dis 2011; 11: 819-824. 
76 World Health Organization. Automated Real-Time Nucleic Acid Amplification Technology for Rapid and Simultaneous Detection of Tuberculosis and Rifampicin Resistance: Xpert MTB/RIF System for the Diagnosis of Pulmonary and Extrapulmonary TB in Adults and Children: Policy Update (WHO/HTM/TB/2013.14). Geneva, WHO, 2013.

77 Tortoli E, Russo C, Piersimoni C, et al. Clinical validation of Xpert MTB/RIF for the diagnosis of extrapulmonary tuberculosis. Eur Respir J 2012; 40: 442-447.

78 Dodd LE, Wilkinson RJ. Diagnosis of pediatric tuberculosis: the culture conundrum. Lancet Infect Dis 2013; 13:3-4.

79 Verhagen LM, Zomer A, Maes M, et al. A predictive signature gene set for discriminating active from latent tuberculosis in Warao Amerindian children. BMC Genomics 2013; 14: 74.

80 Nicol MP, Allen V, Workman L, et al. Urine lipoarabinomannan testing for diagnosis of pulmonary tuberculosis in children: a prospective study. Lancet Glob Health 2014; 2: e278-e284.

81 Kolk A, Hoelscher M, Maboko L, et al. Electronic-nose technology using sputum samples in diagnosis of patients with tuberculosis. J Clin Microbiol 2010; 48: 4235-4238.

82 Amanullah F, Ashfaq M, Khowaja S, et al. High tuberculosis prevalence in children exposed at home to drugresistant tuberculosis. Int J Tuberc Lung Dis 2014; 18: 520-527.

83 Morcos W, Morcos M, Doss S, et al. Drug-resistant tuberculosis in Egyptian children using Etest. Minerva Pediatr 2008; 60: 1385-1392.

84 Esposito S, D’Ambrosio L, Tadolini M, et al. ERS/WHO Tuberculosis Consilium assistance with extensively drugresistant tuberculosis management in a child: case study of compassionate delamanid use. Eur Respir J 2014; 44: 811-815.

85 Ettehad D, Schaaf HS, Seddon JA, et al. Treatment outcomes for children with multidrug-resistant tuberculosis: a systematic review and meta-analysis. Lancet Infect Dis 2012; 12: 449-456.

86 Seddon JA, Hesseling AC, Willemse M, et al. Culture-confirmed multidrug-resistant tuberculosis in children: clinical features, treatment, and outcome. Clin Infect Dis 2012; 54: 157-166.

87 Williams B, Ramroop S, Shah P, et al. Multidrug-resistant tuberculosis in UK children: presentation, management and outcome. Eur Respir J 2013; 41: 1456-1458.

88 Schaaf HS, Marais BJ, Hesseling AC, et al. Surveillance of antituberculosis drug resistance among children from the Western Cape Province of South Africa - an upward trend. Am J Public Health 2009; 99: 1486-1490.

89 World Health Organization. Guidelines for the Programmatic Management of Drug-Resistant Tuberculosis: 2011 Update. Geneva, WHO, 2011

90 Delacourt C. Particularités de la tuberculose chez l'enfant [Specific features of tuberculosis in childhood]. Rev Mal Respir 2011; 28: 529-541.

91 Swingler GH, du Toit G, Andronikou S, et al. Diagnostic accuracy of chest radiography in detecting mediastinal lymphadenopathy in suspected pulmonary tuberculosis. Arch Dis Child 2005; 90: 1153-1156.

92 Hatherill M, Verver S, Mahomed H. Consensus statement on diagnostic end points for infant tuberculosis vaccine trials. Clin Infect Dis 2012; 54: 493-501.

93 Gwee A, Pantazidou A, Ritz N, et al. To x-ray or not to x-ray? Screening asymptomatic children for pulmonary TB: a retrospective audit. Arch Dis Child 2013; 98: 401-404.

94 Goussard P, Gie RP, Kling S, et al. The outcome of infants younger than 6 months requiring ventilation for pneumonia caused by Mycobacterium tuberculosis. Pediatr Pulmonol 2008; 43: 505-510.

95 Arlaud K, Gorincour G, Bouvenot J, et al. Could CT scan avoid unnecessary flexible bronchoscopy in children with active pulmonary tuberculosis? A retrospective study. Arch Dis Child 2010; 95: 125-129.

96 Gupta A, Pauliah S, Urquhart DS, et al. Endobronchial polyp secondary to pulmonary tuberculosis. Arch Dis Child 2009; 94: 230.

97 Goussard P, Gie RP, Kling S, et al. Bronchoscopic assessment of airway involvement in children presenting with clinically significant airway obstruction due to tuberculosis. Pediatr Pulmonol 2013; 48: 1000-1007.

98 Abuogi LL, Mwachari C, Leslie HH, et al. Impact of expanded antiretroviral use on incidence and prevalence of tuberculosis in children with HIV in Kenya. Int J Tuberc Lung Dis 2013; 17: 1291-1297.

99 Venturini E, Turkova A, Chiappini E, et al. Tuberculosis and HIV co-infection in children. BMC Infect Dis 2014; 14: Suppl. 1, S5.

100 Yotebieng M, Van Rie A, Moultrie H, et al. Effect on mortality and virological response of delaying antiretroviral therapy initiation in children receiving tuberculosis treatment. AIDS 2010; 24: 1341-1349.

101 Pefura Yone EW, Evouna Mbarga A, Kuaban C. Impact de l'infection a VIH sur la tuberculose de l'enfant a Yaounde, Cameroun [The impact of HIV infection on childhood tuberculosis in Yaounde, Cameroon]. Rev Mal Respir 2012; 29: 1095-1103.

102 Hailu D, Abegaz WE, Belay M. Childhood tuberculosis and its treatment outcomes in Addis Ababa: a 5-years retrospective study. BMC Pediatr 2014; 14: 61.

103 van Leth F, Kampmann B. Embracing the challenges of HIV-TB co-infection in children. Int J Tuberc Lung Dis 2014; 18: 379.

104 Thee S, Seddon JA, Donald PR, et al. Pharmacokinetics of isoniazid, rifampin, and pyrazinamide in children younger than two years of age with tuberculosis: evidence for implementation of revised World Health Organization recommendations. Antimicrob Agents Chemother 2011; 55: 5560-5567.

105 Schaaf HS, Victor TC, Engelke E, et al. Minimal inhibitory concentration of isoniazid in isoniazid-resistant Mycobacterium tuberculosis isolates from children. Eur J Clin Microbiol Infect Dis 2007; 26: 203-205.

106 Satyanarayana S, Shivashankar R, Vashist RP, et al. Characteristics and programme-defined treatment outcomes among childhood tuberculosis (TB) patients under the National TB Programme in Delhi. PLoS One 2010; 5: e13338.

107 Menon PR, Lodha R, Sivanandan S, et al. Intermittent or daily short course chemotherapy for tuberculosis in children: meta-analysis of randomized controlled trials. Indian Pediatr 2010; 47: 67-73.

108 Chang KC, Leung CC, Grosset J, et al. Treatment of tuberculosis and optimal dosing schedules. Thorax 2011; 66: 997-1007.

109 Ormerod LP. Drug therapy for children with tuberculosis. Arch Dis Child 2012; 97: 1097-1101.

110 Thampi N, Stephens D, Rea E, et al. Unexplained deterioration during antituberculous therapy in children and adolescents: clinical presentation and risk factors. Pediatr Infect Dis J 2012; 31: 129-133.

111 Goussard P, Gie R. Airway involvement in pulmonary tuberculosis. Paediatr Respir Rev 2007; 8: 118-123. 
112 van Dijk JH, Sutcliffe CG, Hamangaba F, et al. Effectiveness of efavirenz-based regimens in young HIV-infected children treated for tuberculosis: a treatment option for resource-limited settings. PLoS One 2013; 8: e55111.

113 Fairlie L, Beylis NC, Reubenson G, et al. High prevalence of childhood multi-drug resistant tuberculosis in Johannesburg, South Africa: a cross sectional study. BMC Infect Dis 2011; 11: 28.

114 Rose PC, Hallbauer UM, Seddon JA, et al. Linezolid-containing regimens for the treatment of drug-resistant tuberculosis in South African children. Int J Tuberc Lung Dis 2012; 16: 1588-1593.

115 Faurholt-Jepsen D, Range N, Praygod G, et al. BCG protects against tuberculosis irrespective of HIV status: a matched case-control study in Mwanza, Tanzania. Thorax 2013; 68: 288-289.

116 Zelner JL, Murray MB, Becerra MC, et al. Bacillus Calmette-Guérin and isoniazid preventive therapy protect contacts of patients with tuberculosis. Am J Respir Crit Care Med 2014; 189: 853-859.

117 Shann F. Nonspecific effects of vaccines and the reduction of mortality in children. Clin Ther 2013; 35: 109-114.

118 Trunz BB, Fine P, Dye C. Effect of BCG vaccination on childhood tuberculous meningitis and miliary tuberculosis worldwide: a meta-analysis and assessment of cost-effectiveness. Lancet 2006; 367: 1173-1180.

119 Dara M, Acosta CD, Rusovich V, et al. Bacille Calmette-Guérin vaccination: the current situation in Europe. Eur Respir J 2014; 43: 24-35.

120 Guthmann JP, Antoine D, Fonteneau L, et al. Assessing BCG vaccination coverage and incidence of paediatric tuberculosis following two major changes in BCG vaccination policy in France. Euro Surveill 2011; 16: 19774.

121 Guthmann JP, Chauvin P, Le Strat Y, et al. Family history of immigration from a tuberculosis endemic country and low family income are associated with a higher BCG vaccination coverage in Ile-de-France region, France. Vaccine 2013; 31: 5666-5671.

122 Montagnani C, Chiappini E, Galli L, et al. Vaccine against tuberculosis: what's new? BMC Infect Dis 2014; 14: Suppl. 1, S2.

123 Ayieko J, Abuogi L, Simchowitz B, et al. Efficacy of isoniazid prophylactic therapy in prevention of tuberculosis in children: a meta-analysis. BMC Infect Dis 2014; 14: 91.

124 Mandalakas AM, Hesseling AC, Gie RP, et al. Modelling the cost-effectiveness of strategies to prevent tuberculosis in child contacts in a high-burden setting. Thorax 2013; 68: 247-255.

125 Zar HJ, Cotton MF, Strauss S, et al. Effect of isoniazid prophylaxis on mortality and incidence of tuberculosis in children with HIV: randomized controlled trial. BMJ 2007; 334: 136.

126 Gray DM, Workman LJ, Lombard CJ, et al. Isoniazid preventive therapy in HIV-infected children on antiretroviral therapy: a pilot study. Int J Tuberc Lung Dis 2014; 18: 322-327.

127 Gray DM, Zar H, Cotton M. Impact of tuberculosis preventive therapy on tuberculosis and mortality in HIVinfected children. Cochrane Database Syst Rev 2009; 1: CD006418.

128 Leung CC, Rieder HL, Lange C, et al. Treatment of latent infection with Mycobacterium tuberculosis: update 2010. Eur Respir J 2011; 37: 690-711.

129 Madhi SA, Nachman S, Violari A, et al. Primary isoniazid prophylaxis against tuberculosis in HIV-exposed children. N Engl J Med 2011; 365: 21-31.

130 Cattamanchi A, Dantes RB, Metcalfe JZ, et al. Clinical characteristics and treatment outcomes of patients with isoniazid-monoresistant tuberculosis. Clin Infect Dis 2009; 48: 179-185.

131 Spyridis NP, Spyridis PG, Gelesme A, et al. The effectiveness of a 9-month regimen of isoniazid alone versus 3- and 4-month regimens of isoniazid plus rifampin for treatment of latent tuberculosis infection in children: results of an 11-year randomized study. Clin Infect Dis 2007; 45: 715-722.

132 Gwee A, Coghlan B, Curtis N. Question 1: what are the options for treating latent TB infection in children? Arch Dis Child 2013; 98: 468-474.

133 Sharma SK, Sharma A, Kadhiravan T, et al. Rifamycins (rifampicin, rifabutin and rifapentine) compared to isoniazid for preventing tuberculosis in HIV-negative people at risk of active TB. Cochrane Database Syst Rev 2013; 7: CD007545.

134 D’Ambrosio L, Dara M, Tadolini M, et al. Tuberculosis elimination: theory and practice in Europe. Eur Respir J 2014; 43: 1410-1420.

135 Voniatis C, Migliori GB, Voniatis M, et al. Tuberculosis elimination: dream or reality? The case of Cyprus. Eur Respir J 2014; 44: 543-546. 